\title{
Therapeutic Administration of Plasminogen Activator Inhibitor-1 Prevents Hypoxic-Ischemic Brain Injury in Newborns
}

\author{
Dianer Yang, ${ }^{1}$ Niza Nemkul, ${ }^{1}$ Ahmed Shereen, ${ }^{2}$ Alice Jone, ${ }^{1}$ R. Scott Dunn, ${ }^{2}$ Daniel A. Lawrence, ${ }^{3}$ Diana Lindquist,${ }^{2}$ and \\ Chia-Yi Kuan ${ }^{1}$ \\ ${ }^{1}$ Division of Developmental Biology and Division of Neurology, ${ }^{2}$ Imaging Research Center, Department of Radiology, Cincinnati Children's Hospital \\ Medical Center, Cincinnati, Ohio 45229, and ${ }^{3}$ Department of Internal Medicine, Division of Cardiovascular Medicine, University of Michigan Medical \\ School, Ann Arbor, Michigan 48109
}

Disruption of the integrity of the blood- brain barrier (BBB) is an important mechanism of cerebrovascular diseases, including neonatal cerebral hypoxia-ischemia (HI). Although both tissue-type plasminogen activator (tPA) and matrix metalloproteinase-9 (MMP-9) can produce $\mathrm{BBB}$ damage, their relationship in neonatal cerebral $\mathrm{HI}$ is unclear. Here we use a rodent model to test whether the plasminogen activator (PA) system is critical for MMP-9 activation and HI-induced brain injury in newborns. To test this hypothesis, we examined the therapeutic effect of intracerebroventricular injection of plasminogen activator inhibitor-1 (PAI-1) in rat pups subjected to unilateral carotid artery occlusion and systemic hypoxia. We found that the injection of PAI-1 greatly reduced the activity of both tPA and urokinase-type plasminogen activator after HI. It also blocked HI-induced MMP-9 activation and BBB permeability at $24 \mathrm{~h}$ of recovery. Furthermore, magnetic resonance imaging and histological analysis showed the PAI-1 treatment reduced brain edema, axonal degeneration, and cortical cell death at $24-48 \mathrm{~h}$ of recovery. Finally, the PAI-1 therapy provided a dose-dependent decrease of brain tissue loss at $7 \mathrm{~d}$ of recovery, with the therapeutic window at $4 \mathrm{~h}$ after the $\mathrm{HI}$ insult. Together, these results suggest that the brain PA system plays a pivotal role in neonatal cerebral $\mathrm{HI}$ and may be a promising therapeutic target in infants suffering hypoxic-ischemic encephalopathy.

\section{Introduction}

Recent studies indicate that neurovascular proteases, including matrix metalloproteases (MMPs) and secreted serine proteases, play a critical role in cerebrovascular diseases (Mun-Bryce and Rosenberg, 1998; Lo et al., 2004). This is because dysregulation of neurovascular proteases degrades the extracellular matrix (ECM) and blood-brain barrier (BBB), leading to brain edema, leukocyte infiltration, and the neuron-matrix detachment. In adult cerebral ischemia, MMP-9 has been suggested to be a promising therapeutic target, because its activity arises early after ischemia and either genetic or pharmacological inhibition of MMP-9 offers brain protection in animal models (Heo et al., 1999; Asahi et al., 2001; Gu et al., 2005). In contrast, the role of MMP-9 in neonatal cerebral hypoxia-ischemia (HI) is unclear. This is because the MMP-9 activity was only detectable at $24 \mathrm{~h}$ of recovery, when some irreversible brain damage has already occurred (Svedin et al., 2007). Thus, it seems unlikely that MMP-9 could be a key initiator of HI brain injury in newborns.

Tissue-type plasminogen activator (tPA) is another important

Received March 7, 2009; revised May 8, 2009; accepted June 1, 2009

This work was supported by National Institutes of Health Grant NS 059668 and the Alzheimer's Association. We thank Drs. Ton DeGrauw and Mark Schapiro for critical reading of this manuscript.

Correspondence should be addressed to Dr. Chia-Yi Kuan, Cincinnati Children's Hospital Medical Center, 3333 Burnet Avenue, Cincinnati, $0 \mathrm{H}$ 45229. E-mail: alex.kuan@cchmc.org.

DOI:10.1523/JNEUROSCI.1117-09.2009

Copyright $\odot 2009$ Society for Neuroscience $\quad$ 0270-6474/09/298669-06\$15.00/0 neurovascular protease that may contribute to neonatal HI brain injury. tPA mainly circulates in the blood but also exists at a low level in the brain parenchyma, which can be induced by excitotoxins (Sappino et al., 1993; Tsirka et al., 1995). tPA directly triggers the opening of $\mathrm{BBB}$ through activation of the latent platelet-derived growth factor $\mathrm{C}$ and elevates the MMP-9 levels after stroke (Yepes et al., 2000; Sumii and Lo, 2002; Wang et al., 2003; Su et al., 2008). Furthermore, we recently reported that cerebral HI produces rapid ( $<1 \mathrm{~h}$ ) and persistent (up to $24 \mathrm{~h}$ ) induction of tPA surrounding the blood vessels and lateral ventricles in newborn brains (Adhami et al., 2008). The early induction of tPA suggests that it may be an initiator of HI brain injury in newborns.

To test this hypothesis, we used intracerebroventricular (ICV) injection of plasminogen activator inhibitor-1 (PAI-1) to block the parenchymal tPA and urokinase-type plasminogen activator ( $\mathrm{uPA}$ ) activity in the Vannucci model of cerebral HI in rat pups and examined its effects on MMP-9 activation and brain damage. Results of these experiments supported our hypothesis and suggested that plasminogen activators (PAs) are potential therapeutic targets in neonatal HI brain injury.

\section{Materials and Methods}

Animal surgery and quantification of tissue loss. Seven-day-old Wistar rat pups were used for the cerebral ischemia-hypoxia model and ICV injection as described previously (Rice et al., 1981; Adhami et al., 2008). The procedures were approved by the Institutional Animal Care and Use 
Committee. The percentage of tissue loss in the cerebral cortex, hippocampus, and striatum was quantified by comparison with individual counterparts on the contralateral hemisphere.

Reagents and biochemistry. Plasminogen/casein zymogram, MMP zymogram, immunoblot, and immunocytochemistry were performed as described previously (Adhami et al., 2008). The procedure of quantifying Evans blue dye extravasation has been described previously (Su et al., 2008). A stable mutant form of human PAI-1 (Berkenpas et al., 1995) was purchased from Molecular Innovations.

Magnetic resonance imaging. All data were acquired on a Bruker BioSpec 7T system with $40 \mathrm{G} / \mathrm{cm}$ gradients using a custom-built 25 $\mathrm{mm}$ single-turn transmit/receive solenoid coil. Animals were scanned in two cohorts, with two each saline-treated or PAI-1-treated animals in the first cohort and three each saline- or PAI-1-treated animals in the second cohort. Animals were brought to the scanner $24 \mathrm{~h}$ after HI induction. They were anesthetized and maintained with $1 \%$ isoflurane in air and kept warm with heated air circulating through the magnet bore. T2weighted anatomical images were acquired using a two-dimensional RARE (rapid acquisition and relaxation enhancement) sequence with an effective echo time of $76.96 \mathrm{~ms}$, repetition time of $1000 \mathrm{~ms}$, field-of-view (FOV) of $19.2 \times 19.2 \mathrm{~mm}^{2}$, and a $256 \times 192$ matrix size. Diffusion tensor images were acquired with a spin echo sequence using an echo time of $21 \mathrm{~ms}$, repetition time of $1100 \mathrm{~ms}$, b-value of 800 , six diffusion directions, FOV of $19.2 \times 19.2$ $\mathrm{mm}^{2}$, and a matrix of $128 \times 128$. For the second cohort, T2 maps were calculated from data acquired with a spin echo sequence using echo times of $20,40,60,80$, and $100 \mathrm{~ms}$ at a repetition time of $1800 \mathrm{~ms}$ with the same FOV and matrix as used for the diffusion scan.

Diffusion data were processed using the Bruker online processing software to calculate apparent diffusion coefficient (ADC) maps and directionally color-encoded (DEC) maps of the fractional anisotropy (FA). T2 maps were calculated using the Bruker online data processing software.

Statistical analysis. Values are represented as mean \pm SD or SEM as indicated. Quantitative data were compared between different groups using Microsoft Excel two-sample (unpaired) $t$ test assuming equal variance.

\section{Results}

Inhibition of plasminogen activators blocks MMP-9 induction after $\mathrm{HI}$

To examine the relationship between PAs and MMP-9 in neonatal cerebral HI, we performed ICV injection of saline or recombinant human PAI-1 (1.9 $\mu$ g per pup) ipsilateral to carotid ligation at the end of a 90 min hypoxic insult $\left(10 \% \mathrm{O}_{2}\right)$ in postnatal day 7 rat pups and collected the brains at 4 or $24 \mathrm{~h}$ recovery for biochemical analysis.

The immunoblot detected the ICV-injected PAI- 1 on the HIchallenged side of brain $(\mathrm{R})$ at 4 but not $24 \mathrm{~h}$ of recovery $(n=4)$ (Fig. 1A,B). The plasminogen/casein zymogram showed a low level of basal tPA activity in the brain, which was increased on the HI-stressed side of brain at both $4 \mathrm{~h}(1.28$-fold increase; $p<0.05)$

D IgG

\section{B 24hr-Recovery}
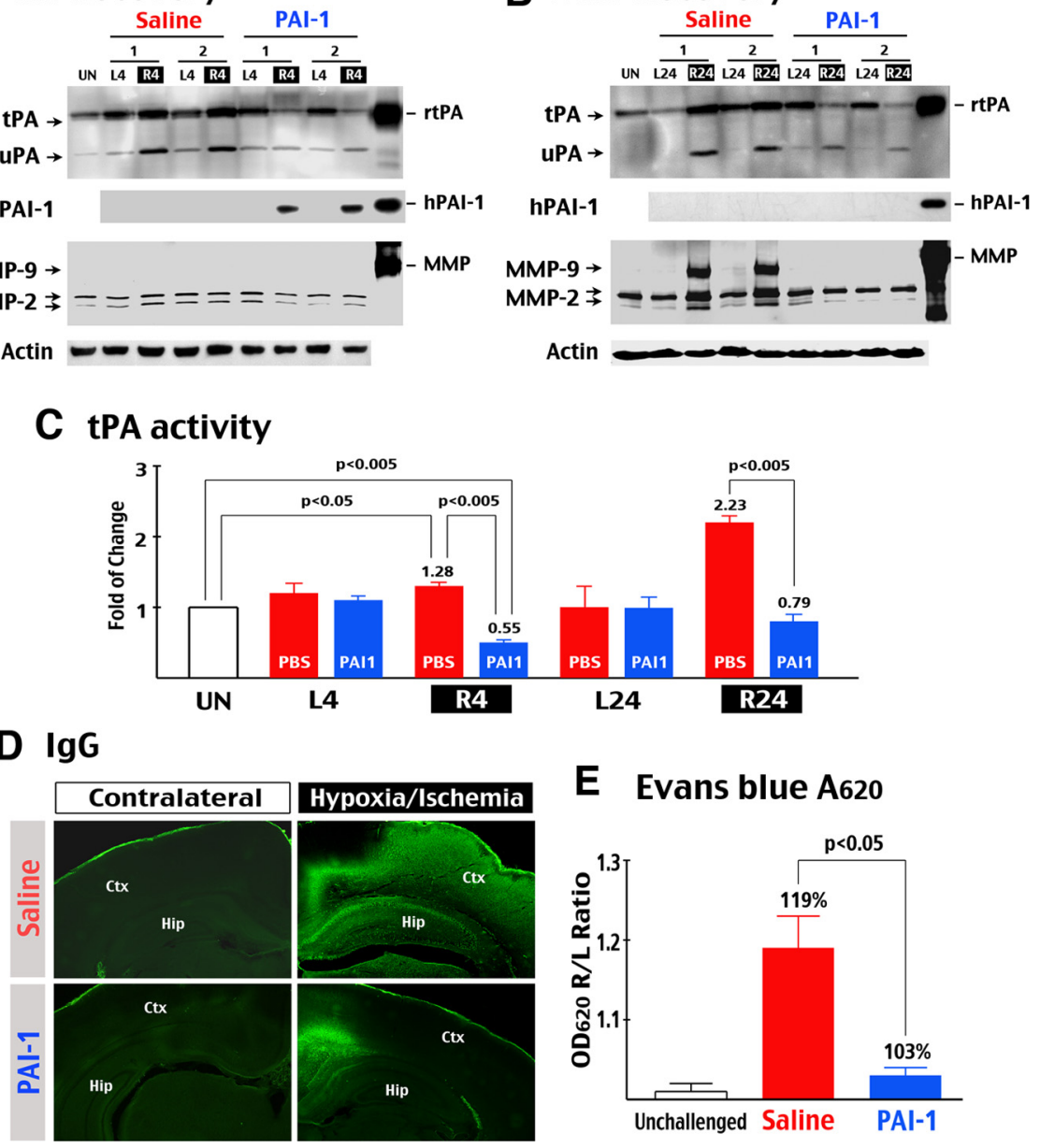

E Evans blue A620

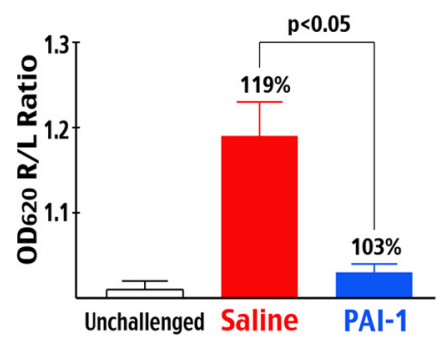

Figure 1. Analysis of PA/MMP activities and BBB permeability within $24 \mathrm{~h}$ after neonatal cerebral hypoxia-ischemia. $A, B$, First panel, Plasminogen/casein zymography showed an increase of both $\mathrm{PA}$ and uPA activities on the $\mathrm{HI}$-challenged side of brain at $4 \mathrm{~h}$ dampened the induction of PAs and MMPs at both 4 and $24 \mathrm{~h}$ of recovery. Second panel, Immunoblot showed retention of the -injected human PAI-1 at $4 \mathrm{~h}$ of recovery, but this was greatly reduced at $24 \mathrm{~h}$ after $\mathrm{HI}$. Third panel, Gelatin zymography showe verified equal loading of a comparable amount of protein in all examined samples. Shown are representative in four to six sets of , Cerebral cortex; Hip, hippocampus. $\boldsymbol{E}$, Quantification of Evans blue extravasation at $24 \mathrm{~h}$ recovery $(n=3)$. Shown in $\boldsymbol{E}$ and $\boldsymbol{G}$ are mean and SE. $p$ value is determined by $t$ test. $L$, Left; $R$, right.

and $24 \mathrm{~h}(2.23$-fold; $p<0.005 ; n=6)$ of recovery after salineinjection (Fig. $1 A-C$ ). Furthermore, the tPA induction was accompanied by an increase of the UPA activity at both time points after HI. In contrast, the induction of MMP-9 and MMP-2 was only detectable at $24 \mathrm{~h}$ but not $4 \mathrm{~h}$ of recovery. The observed timings of MMP-9 induction were consistent with a previous report (Svedin et al., 2007).

However, in contrast to saline injection, the PAI-1 injection greatly reduced the tPA activity at both $4 \mathrm{~h}(0.55$-fold of the basal level) and $24 \mathrm{~h}$ (0.79-fold of the basal level) of recovery $(p<$ 0.005 compared with saline-injected animals; $n=6$ for each time point) (Fig. $1 A-C$ ). The HI-induced uPA activity was also diminished to $57-78 \%$ of those in saline-injected animals. Furthermore, the PAI-1 treatment almost completely blocked the HIinduced MMP-9 and MMP-2 activation at $24 \mathrm{~h}$ of recovery (Fig. $1 B)$. These results indicated that activation of the PA system precedes and is required for MMP-9 induction in neonatal cerebral HI. 


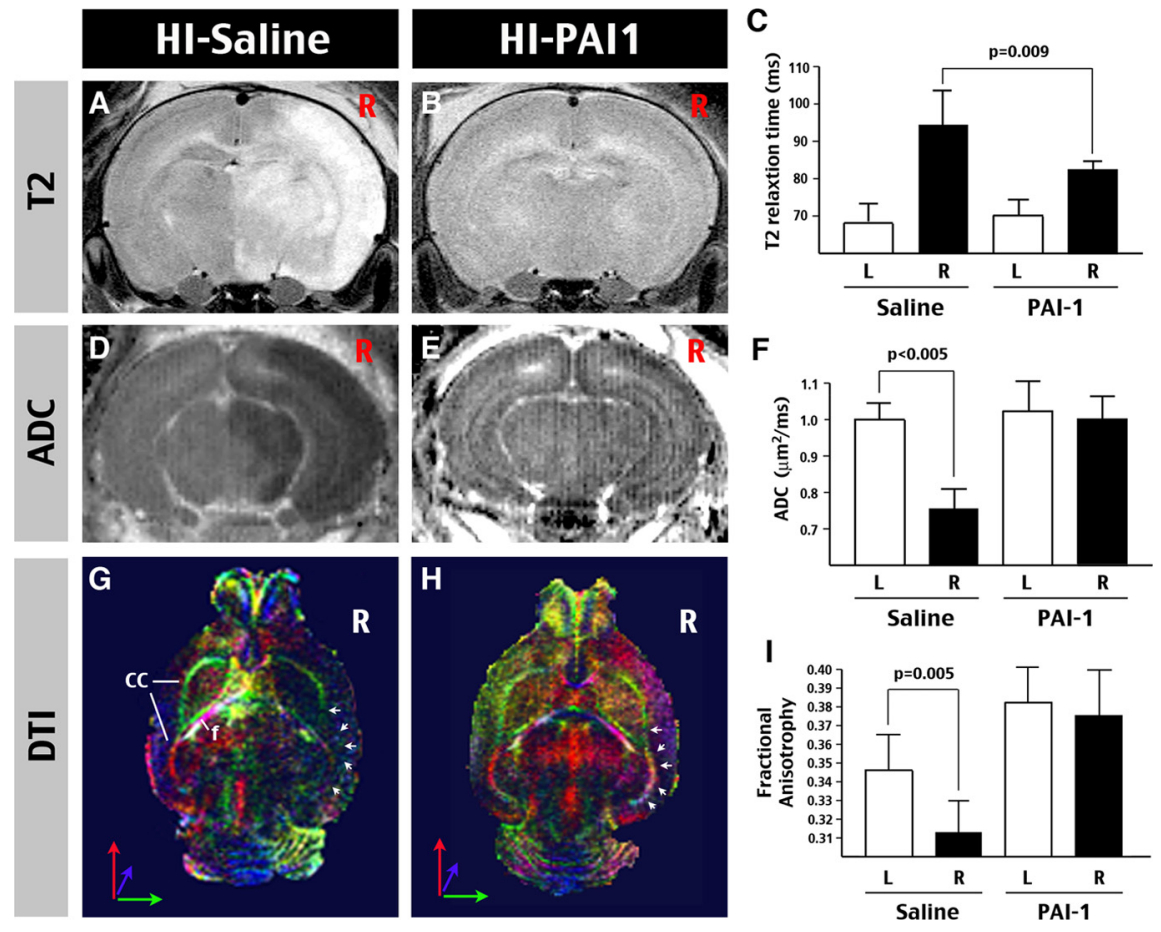

Figure 2. MRI at $24 \mathrm{~h}$ after hypoxia-ischemia. $\boldsymbol{A}, \boldsymbol{B}, \mathrm{T}$-weighted image of $\mathrm{HI}$-challenged and saline-treated $(\boldsymbol{A})$ or PAI-1treated $(\boldsymbol{B})$ brain at $24 \mathrm{~h}$. Saline-treated animals show a large area of increased T2 signal intensity on the lesion (R) side. $\boldsymbol{C}$ Quantification showed T2 signal increased from $68 \mathrm{~ms}$ on the contralateral side to $95 \mathrm{~ms}$ on the ipsilateral side in saline-injected animals. By comparison, 12 signal increased from 72 ms on contralateral side to 83 ms on ipsilateral side in PAI-1-injected animals. The difference of increase from the contralateral side was significant $(p=0.009$ by $t$ test; $n=3$ ). $\boldsymbol{D}, \boldsymbol{E}$, Diffusion-weighted image of saline-treated $(\boldsymbol{D})$ or PAl-1-treated $(\boldsymbol{E})$ brain at $24 \mathrm{~h}$. Note the large area of decreased ADC on the lesion side of saline-injected rat brains. $\boldsymbol{F}$, Quantification showed a significant reduction of $A D C$ in saline-injected animals ( $p<0.005$ by $t$ test; $n=3) . \boldsymbol{G}, \boldsymbol{H}$, DTI and quantitative DEC map at $24 \mathrm{~h}$. f, Fibrim. Note the absence of fibrim and posterior CC (arrows) in saline-injected (G) but not in PAl-1-injected $(\boldsymbol{H})$ animal brain. $\boldsymbol{I}$, Quantification showed FA dropped from 0.347 on the contralateral side to 0.314 on the ipsilateral side in saline-injected rats ( $p=0.005$ by $t$ test; $n=5)$. There was no obvious difference of FA on contralateral side $(0.383 \pm 0.018$, mean \pm SEM) and ipsilateral side $(0.378 \pm 0.020)$ in PAl-1-treated animals $(n=5)$. L, Left; $R$, right.

\section{PAI-1 therapy protects against $\mathrm{HI}$-induced $\mathrm{BBB}$ permeability} and brain edema

Because tPA and MMP are both potent neurovascular proteases, we next examined the effect of PAI-1 therapy on HI-induced BBB permeability at $24 \mathrm{~h}$ of recovery. We found that saline-injected animals consistently displayed Ig extravasation over a larger area on the HI-stressed side of brain than PAI-1-injected animals ( $n=$ 4) (Fig. 1D). In addition, the PAI-1 therapy decreased the extent of HI-induced Evans blue dye extravasation from 19 to 3\% over the contralateral side of brain $(p<0.05 ; n=3)$ (Fig. $1 E$ ). These results suggest more severe $\mathrm{BBB}$ damage in saline-injected animals.

Next, we used a 7 tesla magnetic resonance imaging (MRI) system to examine the effect of PAI-1 therapy in preserving the $\mathrm{BBB}$ integrity at $24 \mathrm{~h}$ after HI. The MRI study showed a large area of increased T2 signal-an indication of water accumulation-on the ipsilateral hemisphere in saline-injected but not PAI-treated animals (Fig. 2A,B). Similarly, a greater increase in $\mathrm{T} 2$ relaxation time over the contralateral side ( 68 to $95 \mathrm{~ms} ; 37.5 \%$ increase) was found in saline-treated animals than in PAI-1 treated animals ( 71 to $82 \mathrm{~ms} ; 15.1 \%$ increase) $(p<0.05$ comparing the percentage increase from the contralateral side; $n=3$ ) (Fig. 2C).

The saline-injected animals also exhibited a large area of reduced ADC - a sign of diffusion-restricted intracellular space or tortuous extracellular pathway and generally used as an indicator of cytotoxic edema (Moseley et al., 1990)—on the ipsilateral hemisphere (Fig. 2D). Quantification showed that ADC dropped from $1.005 \pm$ $0.047 \mu \mathrm{m}^{2} / \mathrm{ms}$ on the contralateral side to $0.755 \pm 0.064$ on the HI-challenged side in saline-injected animals $(p<0.005 ; n=3)$ (Fig. $2 F$ ). In contrast, the reduction of ADC after HI was insignificant in PAI-1 animals $(1.032 \pm 0.047$ on the contralateral side and $1.026 \pm 0.051$ on the ipsilateral side) $(n=3)$ (Fig. $2 E, F)$. Together, these results indicate that inhibition of plasminogen activators reduces $\mathrm{HI}$ induced $\mathrm{BBB}$ damage and brain edema in newborns.

\section{PAI-1 therapy protects against HI- induced white-matter damage and cortical degeneration}

Next, we used diffusion tensor imaging (DTI) to test whether ICV injection of PAI-1 lessens the HI-induced whitematter injury in this model, which is commonly used as an experimental paradigm of periventricular leukomalacia in infants (Volpe, 2008). We found that salineinjected rats showed $\sim 10 \%$ reduction of FA in the corpus callosum (CC) at $24 \mathrm{~h}$ of recovery $(p=0.005 ; n=5$ ) (Fig. $2 I$ ). In contrast, PAI-1-treated animals showed little reduction of FA in the CC (Fig. 2I). Furthermore, DEC map of DTI-a method to highlight the orientation of anisotropic tissues (Chahboune et al., 2007) — frequently showed partial absence of the corpus callosum tract in salineinjected animals (Fig. $2 G$, arrows) but not in PAI-1-treated animals (Fig. $2 \mathrm{H}$ ). These results suggest that PAI-1 therapy ameliorates the white-matter injury in neonatal cerebral HI.

To confirm the efficacy of PAI-1 therapy against HI-induced axonal damage, we examined the histology of saline- or PAI-1treated animals at $48 \mathrm{~h}$ recovery $(n=9$ for each). This analysis showed that almost all saline-injected animals had brain damage, ranging from massive cystic degeneration to multiple columnar lesions in the cerebral cortex (Fig. $3 B$, arrows), associated with positive terminal deoxynucleotidyl transferase-mediated biotinylated UTP nick end labeling (TUNEL) stain (Fig. 3E) and a swollen defasciculated CC (outlined in Fig. $3 B$ ). In contrast, the PAI-1-treated animals exhibited milder CC swelling and only isolated spots of TUNEL stain (Fig. 3C,F). No apparent lesion was found on the contralateral side of brain in either saline- or PAI-1-injected animals (Fig. $3 A, D$ ).

In addition, the myelin basic protein (MBP)-positive oligodendrocyte processes in the CC were more fragmented and the soma less obvious in saline-injected animals (Fig. $3 H, K$ ) when compared with those on the contralateral side (Fig. 3G,J) or the PAI-1-injected animals (Fig. 3I,L). Furthermore, the salineinjected animals tend to have more OX42-positive macrophages in the CC on the HI-challenged side of the brain than the contralateral side or PAI-1-treated animals (Fig. 3M-O). These results suggest that PAI-1 therapy decreases HI-induced whitematter injury partially through blocking the brain infiltration of inflammatory cells. 
PAI-1 therapy protects against HIinduced brain damage

Finally, we examined the therapeutic effect of PAI-1 injection at $7 \mathrm{~d}$ of recovery. The brains of saline- or PAI-1-treated animals in each experiment were photographed for records (examples in Fig. $4 A, B$ ), serial sectioned, and Nissl stained (Fig. $4 C, D$ ). The extent of brain injury was quantified as the percentage of tissue loss, compared with their counterparts on the contralateral hemisphere, in the cerebral cortex, hippocampus, and striatum. For comparison, we have shown previously that ICV injection of $\alpha 2$-antiplasmin, an inhibitor of one of the many downstream effectors of PAs, produced a maximal 55\% reduction of tissue loss if it was administered within $2 \mathrm{~h}$ after HI (Adhami et al., 2008).

For deriving the dose-response curve (Fig. $4 E$ ), saline or a varying dose of PAI-1 $(0.95-3.8 \mu \mathrm{g})$ was injected within $10 \mathrm{~min}$ after the $\mathrm{HI}$ insult. The extent of tissue loss in saline-injected animals was $67 \pm 3 \%$ (mean $\pm \mathrm{SE}$ ) in the cerebral cortex, $64 \pm$ $2 \%$ in the hippocampus, and $53 \pm 3 \%$ in the striatum $(n=29)$. ICV injection of PAI-1 in all doses provided a significant reduction of tissue loss in all three regions ( $n=11-29$ for each dose; $p<0.001)$. PAI-1 at $1.9 \mu \mathrm{g}$ appeared to have the best protection, which remarkably decreased tissue loss to $4 \pm 1 \%$ in the cerebral cortex and $5 \pm 1 \%$ in the hippocampus or striatum when compared with contralateral counterparts $(n=29)$.

To determine the therapeutic window (Fig. $4 F), 1.9 \mu$ g of PAI- 1 was injected at 1 , 2 , or 4 h recovery $(n=10$ for each), and the effects were compared with those after immediate post-HI injection. We found that delayed injection of PAI- 1 at 1 or $2 \mathrm{~h}$ still provided a significant protection in all three regions when compared with saline injection $(p<0.001)$. Even $4 \mathrm{~h}$ delayed injection of PAI-1 decreased tissue loss significantly: $45 \pm 4 \%$ in the cerebral cortex $(p=0.01), 42 \pm 3 \%$ in the hippocampus $(p=0.02)$, and $34 \pm$ $3 \%$ in the striatum $(p=0.09)$. Together, these results suggest that inhibition of PAs is a powerful brain-protection strategy in neonatal $\mathrm{HI}$.

\section{Discussion}

PA is upstream of MMP in neonatal HI brain injury

Uninhibited extracellular protease activity in the "neurovascular unit" - a conceptual entity that comprises neurons, microvessels, and the supporting glial cells- has an important pathogenic role in cerebrovascular disorders (Mun-Bryce and Rosenberg, 1998; Iadecola, 2004; Lo et al., 2004). These "neurovascular proteases" include MMPs, plasmin, plasminogen activators, and thrombin. Among them, MMP-9 and tPA have been discussed as potential therapeutic targets in adult ischemic stroke, because they both show early induction after in-
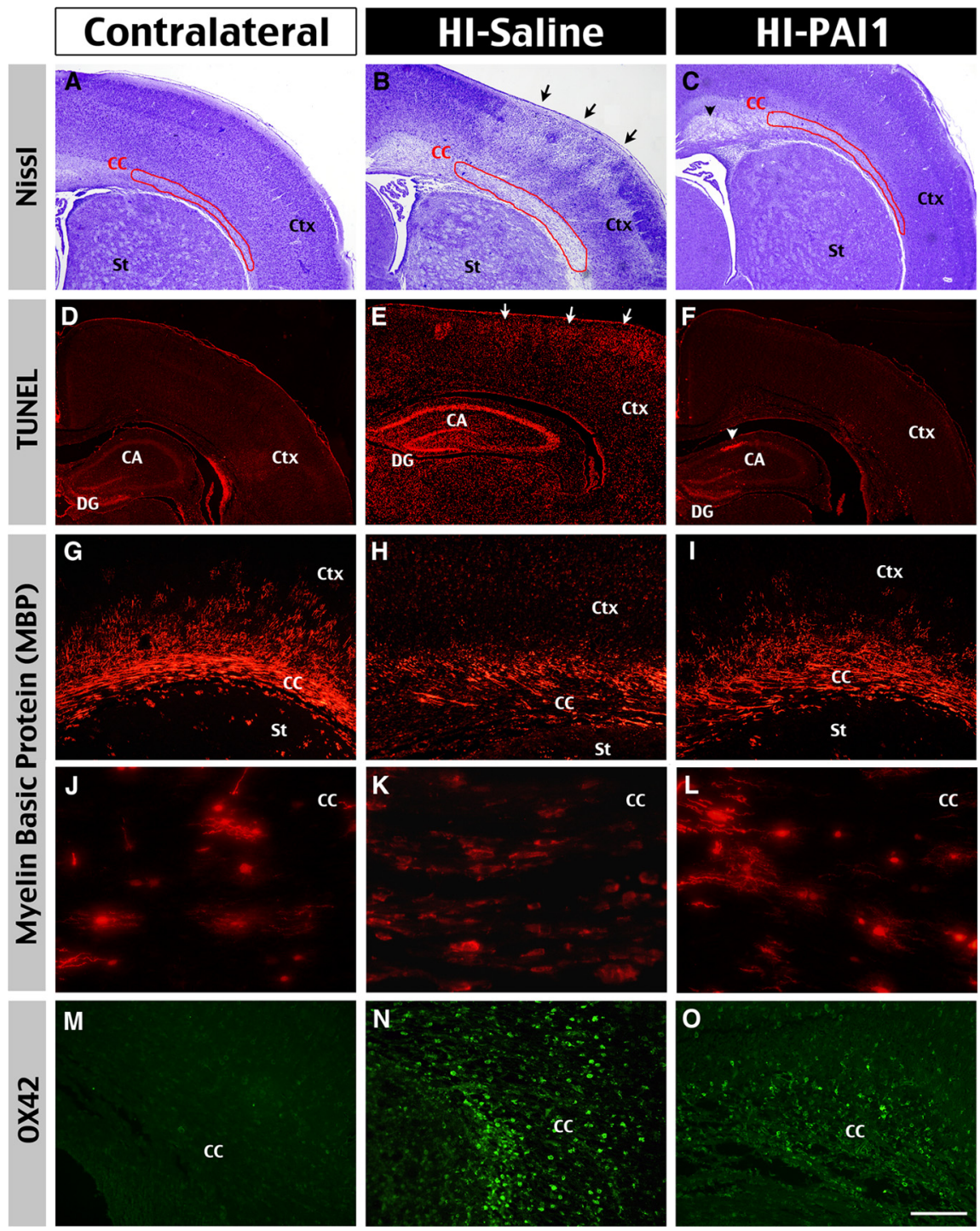

Figure 3. Histological analysis at 48 h after hypoxia-ischemia. Nissl stain $(\boldsymbol{A}-\boldsymbol{C})$, TUNEL stain $(\boldsymbol{D}-\boldsymbol{F})$, anti-MBP stain $(\mathbf{G}-\boldsymbol{L})$, and anti-0X42 stain (M-0) of the contralateral side (left column), HI-challenged and saline-injected (middle column), and HI/PAI-1injected (right column) of rat brains at $48 \mathrm{~h}$ of recovery. Shown are representative images in nine animals of each group. Note the $\boldsymbol{K})$ of oligodendrocytes and infiltration of OX42-positive macrophage in the $C(\boldsymbol{N})$. The PAl-1-treated brains showed localized CC swelling and TUNEL signals (arrowheads in $\boldsymbol{C}, \boldsymbol{F})$. Ctx, Cerebral cortex; St, striatum; CA, Ammon's horn of hippocampus; DG, dentate gyrus. Scale bar: $\mathbf{A}-\boldsymbol{F}, 250 \mu \mathrm{m} ; \mathbf{G}-\mathbf{I}, 100 \mu \mathrm{m} ; \boldsymbol{J}-\mathbf{L}, 20 \mu \mathrm{m} ; \boldsymbol{M}-\mathbf{0}, 50 \mu \mathrm{m}$.

jury, and either genetic or pharmacological inhibition of their activities offers protection in animal models (Wang et al., 1998; Nagai et al., 1999; Yepes et al., 2000; Asahi et al., 2001; Cinelli et al., 2001; Gu et al., 2005). However, little is known about the relative roles of $\mathrm{PAA}$ and MMPs in neonatal cerebral HI.

In this context, the present study revealed a surprising, causal relationship between PA and MMP in neonatal cerebral HI. We have shown previously that $\mathrm{HI}$ induces rapid tPA and uPA activity within $4 \mathrm{~h}$ in the newborn brain (Adhami et al., 2008), whereas the induction of MMP-9 occurs at $24 \mathrm{~h}$ after HI (Svedin et al., 2007). Here, we further demonstrate that early inhibition of PAs after the HI insult is sufficient to prevent the subsequent MMP activation in newborn brains. These results suggest that PA is upstream of MMP in this experimental paradigm. 

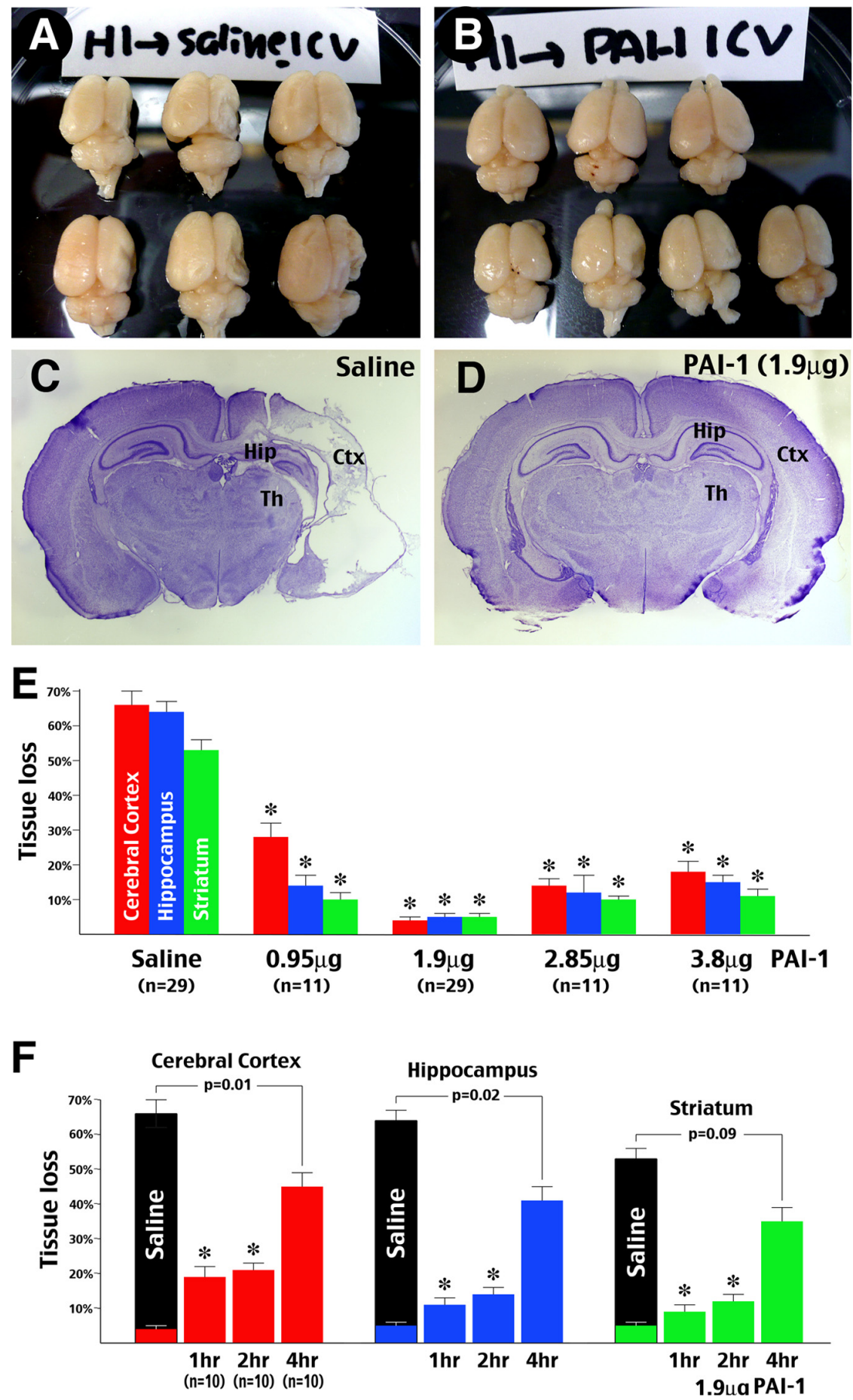

Figure 4. Therapeutic efficacy of PAl-1 evaluated at $7 \mathrm{~d}$ after hypoxia-ischemia. $\boldsymbol{A}, \boldsymbol{B}$, Examples of HI-challenged and salineinjected $(\boldsymbol{A})$ or PAl-1-treated $(\boldsymbol{B})$ rat brains at $7 \mathrm{~d}$ of recovery. Note that the majority of saline-injected animals showed significant tissue loss on the right side of the brain. $\boldsymbol{C}, \boldsymbol{D}$, Examples of Nissl-stained brain sections of saline-injected (C) or PAl-1-treated (D) rat brains. Note prominent cystic degeneration in the cerebral cortex of saline-treated brains. Ctx, cerebral cortex; Hip, hippocampus; Th, thalamus. $\boldsymbol{E}$, Dose-response curve of tissue loss compared with counterparts on the contralateral hemisphere in the cerebral cortex (red), hippocampus (blue), and striatum (green) of animals receiving saline or PAl-1 injection immediately after the $\mathrm{HI}$ insult. ${ }^{*} p<0.001$ compared with saline-injected rats $(n=11-29)$. $\boldsymbol{F}$, Therapeutic window of $1.9 \mu \mathrm{g}$ of PAl-1 injected at 1,2, or $4 \mathrm{~h}$ after the cerebral $\mathrm{HI}$ insult. ${ }^{*} p<0.001$ compared with the saline-injected animals. The $p$ values by $t$ test for $4 \mathrm{~h}$ delayed injection compared with saline-injected animals were indicated.

A pivotal role of PA in neonatal $\mathrm{HI}$ brain injury

Furthermore, the present study shows that ICV injection of PAI-1 provided a greater reduction of injury and a longer therapeutic window against neonatal HI brain injury than targeting the plasmin or MMP-9 activities, as shown in previous studies (Svedin et al., 2007; Adhami et al., 2008). This pattern of differential therapeutic effects suggests a model in which PA (or tPA alone) plays a key initiator role in neonatal HI brain injury (supplemental Fig. 1, available at www.jneurosci.org as supplemental material). Specifically, the HI-induced tPA may directly trigger the opening of $\mathrm{BBB}$ to allow blood-borne cells to enter the brain parenchyma, leading to an increased inflammatory response and MMP activity (McColl et al., 2008; Su et al., 2008). tPA can also function as a cytokine to stimulate microglia to secrete more MMPs (Siao and Tsirka, 2002). Finally, the tPA-converted plasmin is a broad-spectrum protease that can degrade many constituents of the ECM and BBB, which may in turn amplify MMP activation as a secondary response (Tsirka et al., 1995). Thus, the experimental data and our hypothesis indicate that MMPs are downstream of the tPA toxicity in neonatal HI brain injury. Nevertheless, because MMPs are potent collagenases capable of causing severe damage to the vascular wall, their activation may accelerate a transformation from transient to sustained BBB disruption (del Zoppo et al., 2007; McColl et al., 2008).

Together, results from the present study suggest that inhibition of the PA system is a more effective strategy of brain protection against neonatal $\mathrm{HI}$ than targeting any of the downstream effectors. Although the toxicity of the PA system is mostly attributed to $\mathrm{PAA}$, future studies are warranted to compare the roles of tPA and uPA in this pathological process.

\section{$\mathrm{PA}$ as potential therapeutic targets in neonatal hypoxic-ischemic encephalopathy}

The experimental paradigm used in the present study (the Vannucci model) is a popular model of neonatal hypoxic-ischemic encephalopathy (HIE) (Rice et al., 1981). HIE is an important cause of perinatal mortality and permanent neurological morbidities, including cerebral palsy and mental retardation, but there is no specific medication against HIE in the current medical practice (Ferriero, 2004). In this context, our findings may have important clinical implications because they show that inhibition of the parenchymal PAs is a powerful strategy of brain protection in the rodent model of HIE. Although additional studies are need to test the efficacy of PAI- 1 therapy in other experimental models and in higher animal species (Derrick et al., 2004), the present results suggest the rationale for a new brain protection strategy in HIE. 
In conclusion, we suggest that future studies are warranted to examine whether infants diagnosed with HIE or at a high risk of cerebral palsy have greater levels of tPA and plasmin in the brain or the CSF. If so, inhibition of the parenchymal PA system may be a promising new therapy to overcome this devastating perinatal disorder.

\section{References}

Adhami F, Yu D, Yin W, Schloemer A, Burns KA, Liao G, Degen JL, Chen J, Kuan CY (2008) Deleterious effects of plasminogen activators in neonatal cerebral hypoxia-ischemia. Am J Pathol 172:1704-1716.

Asahi M, Wang X, Mori T, Sumii T, Jung JC, Moskowitz MA, Fini ME, Lo EH (2001) Effects of matrix metalloproteinase-9 gene knock-out on the proteolysis of blood-brain barrier and white matter components after cerebral ischemia. J Neurosci 21:7724-7732.

Berkenpas MB, Lawrence DA, Ginsburg D (1995) Molecular evolution of plasminogen activator inhibitor-1 functional stability. EMBO J 14:2969-2977.

Chahboune H, Ment LR, Stewart WB, Ma X, Rothman DL, Hyder F (2007) Neurodevelopment of C57B/L6 mouse brain assessed by in vivo diffusion tensor imaging. NMR Biomed 20:375-382.

Cinelli P, Madani R, Tsuzuki N, Vallet P, Arras M, Zhao CN, Osterwalder T, Rülicke T, Sonderegger P (2001) Neuroserpin, a neuroprotective factor in focal ischemic stroke. Mol Cell Neurosci 18:443-457.

del Zoppo GJ, Milner R, Mabuchi T, Hung S, Wang X, Berg GI, Koziol JA (2007) Microglial activation and matrix protease generation during focal cerebral ischemia. Stroke 38:646-651.

Derrick M, Luo NL, Bregman JC, Jilling T, Ji X, Fisher K, Gladson CL, Beardsley DJ, Murdoch G, Back SA, Tan S (2004) Preterm fetal hypoxiaischemia causes hypertonia and motor deficits in the neonatal rabbit: a model for human cerebral palsy? J Neurosci 24:24-34

Ferriero DM (2004) Neonatal brain injury. N Engl J Med 351:1985-1995.

Gu Z, Cui J, Brown S, Fridman R, Mobashery S, Strongin AY, Lipton SA (2005) A highly specific inhibitor of matrix metalloproteinase-9 rescues laminin from proteolysis and neurons from apoptosis in transient focal cerebral ischemia. J Neurosci 25:6401-6408.

Heo JH, Lucero J, Abumiya T, Koziol JA, Copeland BR, del Zoppo GJ (1999) Matrix metalloproteinases increase very early during experimental focal cerebral ischemia. J Cereb Blood Flow Metab 19:624-633.

Iadecola C (2004) Neurovascular regulation in the normal brain and in Alzheimer's disease. Nat Rev Neurosci 5:347-360.

Lo EH, Broderick JP, Moskowitz MA (2004) tPA and proteolysis in the neurovascular unit. Stroke 35:354-356.

McColl BW, Rothwell NJ, Allan SM (2008) Systemic inflammation alters the kinetics of cerebrovascular tight junction disruption after experimental stroke in mice. J Neurosci 28:9451-9462.

Moseley ME, Cohen Y, Mintorovitch J, Chileuitt L, Shimizu H, Kucharczyk J Wendland MF, Weinstein PR (1990) Early detection of regional cerebral ischemia in cats: comparison of diffusion- and T2-weighted MRI and spectroscopy. Magn Reson Med 14:330-346.

Mun-Bryce S, Rosenberg GA (1998) Matrix metalloproteinases in cerebrovascular disease. J Cereb Blood Flow Metab 18:1163-1172.

Nagai N, De Mol M, Lijnen HR, Carmeliet P, Collen D (1999) Role of plasminogen system components in focal cerebral ischemic infarction: a gene targeting and gene transfer study in mice. Circulation 99:2440-2444.

Rice JE 3rd, Vannucci RC, Brierley JB (1981) The influence of immaturity on hypoxic-ischemic brain damage in the rat. Ann Neurol 9:131-141.

Sappino AP, Madani R, Huarte J, Belin D, Kiss JZ, Wohlwend A, Vassalli JD (1993) Extracellular proteolysis in the adult murine brain. J Clin Invest 92:679-685.

Siao CJ, Tsirka SE (2002) Tissue plasminogen activator mediates microglial activation via its finger domain through annexin II. J Neurosci 22:3352-3358.

Su EJ, Fredriksson L, Geyer M, Folestad E, Cale J, Andrae J, Gao Y, Pietras K, Mann K, Yepes M, Strickland DK, Betsholtz C, Eriksson U, Lawrence DA (2008) Activation of PDGF-CC by tissue plasminogen activator impairs blood-brain barrier integrity during ischemic stroke. Nat Med 14:731-737.

Sumii T, Lo EH (2002) Involvement of matrix metalloproteinase in thrombolysis-associated hemorrhagic transformation after embolic focal ischemia in rats. Stroke 33:831-836.

Svedin P, Hagberg H, Sävman K, Zhu C, Mallard C (2007) Matrix metalloproteinase- 9 gene knock-out protects the immature brain after cerebral hypoxia-ischemia. J Neurosci 27:1511-1518.

Tsirka SE, Gualandris A, Amaral DG, Strickland S (1995) Excitotoxininduced neuronal degeneration and seizure are mediated by tissue plasminogen activator. Nature 377:340-344.

Volpe JJ (2008) Neurology of the newborn, Ed 5. Philadelphia: Saunders Elsevier.

Wang X, Lee SR, Arai K, Lee SR, Tsuji K, Rebeck GW, Lo EH (2003) Lipoprotein receptor-mediated induction of matrix metalloproteinase by tissue plasminogen activator. Nat Med 9:1313-1317.

Wang YF, Tsirka SE, Strickland S, Stieg PE, Soriano SG, Lipton SA (1998) Tissue plasminogen activator (tPA) increases neuronal damage after focal cerebral ischemia in wild-type and tPA-deficient mice. Nat Med 4:228-231.

Yepes M, Sandkvist M, Wong MK, Coleman TA, Smith E, Cohan SL, Lawrence DA (2000) Neuroserpin reduces cerebral infarct volume and protects neurons from ischemia-induced apoptosis. Blood 96:569-576. 